\section{Michigan Technological

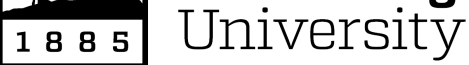

Michigan Technological University Digital Commons @ Michigan Tech

\title{
Environmental Impacts of Distributed Manufacturing from 3-D Printing of Polymer Components and Products
}

\author{
Megan Kreiger \\ Michigan Technological University \\ Joshua M. Pearce \\ Michigan Technological University
}

Follow this and additional works at: https://digitalcommons.mtu.edu/materials_fp

\section{Recommended Citation}

Kreiger, M., \& Pearce, Joshua M. (2013). Environmental impacts of distributed manufacturing from 3-D printing of polymer components and products C3 - Materials Research Society Symposium Proceedings. MRS Online Proceedings Library, 1492(mrsf12-1492-g01-02), 85-90. http://digitalcommons.mtu.edu/ materials_fp/52 
Mater. Res. Soc. Symp. Proc. Vol. 1492 @ 2013 Materials Research Society

DOI: 10.1557/opl.2013.319

\title{
Environmental Impacts of Distributed Manufacturing from 3-D Printing of Polymer Components and Products
}

\author{
Megan Kreiger $^{1}$ and Joshua M. Pearce ${ }^{1,2}$ \\ ${ }^{1}$ Department of Materials Science \& Engineering, Michigan Technological University, \\ Houghton, MI, United States. \\ ${ }^{2}$ Department of Electrical \& Computer Engineering, Michigan Technological University, \\ Houghton, MI, United States.
}

\begin{abstract}
Although additive layer manufacturing is well established for rapid prototyping the low throughput and historic costs have prevented mass-scale adoption. The recent development of the RepRap, an open source self-replicating rapid prototyper, has made low-cost 3-D printers readily available to the public at reasonable prices $(<\$ 1,000)$. The RepRap (Prusa Mendell variant) currently prints 3-D objects in a 200x200x140 square millimeters build envelope from acrylonitrile butadiene styrene (ABS) and polylactic acid (PLA). ABS and PLA are both thermoplastics that can be injection-molded, each with their own benefits, as ABS is rigid and durable, while PLA is plant-based and can be recycled and composted. The melting temperature of ABS and PLA enable use in low-cost 3-D printers, as these temperature are low enough to use in melt extrusion in the home, while high enough for prints to retain their shape at average use temperatures. Using 3-D printers to manufacture provides the ability to both change the fill composition by printing voids and fabricate shapes that are impossible to make using tradition methods like injection molding. This allows more complicated shapes to be created while using less material, which could reduce environmental impact.

As the open source 3-D printers continue to evolve and improve in both cost and performance, the potential for economically-viable distributed manufacturing of products increases. Thus, products and components could be customized and printed on-site by individual consumers as needed, reversing the historical trend towards centrally mass-manufactured and shipped products. Distributed manufacturing reduces embodied transportation energy from the distribution of conventional centralized manufacturing, but questions remain concerning the potential for increases in the overall embodied energy of the manufacturing due to reduction in scale. In order to quantify the environmental impact of distributed manufacturing using 3-D printers, a life cycle analysis was performed on a plastic juicer. The energy consumed and emissions produced from conventional large-scale production overseas are compared to experimental measurements on a RepRap producing identical products with ABS and PLA. The results of this LCA are discussed in relation to the environmental impact of distributed manufacturing with 3-D printers and polymer selection for 3-D printing to reduce this impact. The results of this study show that distributed manufacturing uses less energy than conventional manufacturing due to the RepRap's unique ability to reduce fill composition. Distributed manufacturing also has less emissions than conventional manufacturing when using PLA and when using ABS with solar photovoltaic power. The results of this study indicate that opensource additive layer distributed manufacturing is both technically viable and beneficial from an ecological perspective.
\end{abstract}




\section{INTRODUCTION}

Since the dawn of the industrial age, manufacturing has been about internally maximizing profit. This search for maximum profit created a general trend in towards large-scale manufacturing in low-labor cost countries, especially for inexpensive plastic products [1-3]. Recently, several open-source (OS) models of commercial rapid prototypers have been developed [4], which offer an alternative model of low-cost production. Historically, commercial rapid prototypers or 3-D printers use additive layer manufacturing (AM) to accurately fabricate custom or generic products out of various materials including plastic, metal or ceramics. The technological development of 3-D printers has been substantial, which has benefited many industries [5-9]. However, the costs of 3-D printers have historically been too expensive to be feasible for distributed or home-based manufacturing. With the introduction of the RepRap, an OS 3-D printer that can be built for under $\$ 500$, this has changed. The RepRap, has opened the door of AM to a wide range of potential users due to cost and simplicity [10] and made distributed small-scale production technically feasible [11].

The RepRap has a $200 \mathrm{~mm}$ x $200 \mathrm{~mm}$ x 140mm (height) build envelope and can currently print using various plastics. The plastics typically used are acrylonitrile butadiene styrene (ABS) and polylactic acid (PLA). ABS and PLA are both thermoplastics that can be injection-molded, each with their own benefits, as ABS is rigid and durable, while PLA is plant-based and can be recycled and composted. The melting temperatures of PLA and ABS allow for safe extrusion, while being high enough to ensure shape retention. Distributed recycling is also being developed to recycle post-consumer products into filament for a 3-D printer, which could further reduce cost and resources required for distributed manufacturing [12].

The use of 3-D printers allows for previously impossible shapes under conventional manufacturing methods (e.g. injection molding) along with the ability to manipulate the inside of an object in multiple ways, such as, fill composition or adding internal parts. The ability to manipulate shapes internally during production has the potential to reduce additional machining during processing. Holes, voids, and other needs within an object that were impossible using methods similar to injection molding previously had to be done using tools, such as, drill presses. These steps are now able to be created during the design step and automatically produced using the RepRap. The ability to change fill composition allows more complicated shapes to be produced with structural integrity with the use of less material. This property combined with the reduction in embodied energy of transportation made available by distributed manufacturing allow for the possibility that it could be less energy and emission intensive than conventional manufacturing. However, questions remain about the environmental benefits of distributed manufacturing due the potential for increases in the overall embodied energy of the manufacturing due to reduction in scale.

This preliminary study explores these questions by probing the technical potential of using a distributed network of RepRaps to produce goods. A life cycle analysis (LCA) of energy consumption and emissions is performed for distributed manufacturing using RepRaps and compared to conventional manufacturing overseas. These results are discussed to draw conclusions about the viability of distributed manufacturing.

\section{METHODS}

The RepRap (Prusa Mendell variant) was used to print all product/product components using 
both ABS and PLA (Fig. 1) using 15\% fill in order to reduce the amount of plastic used to produce it while maintaining the necessary functional mechanical strength. The ABS extruder temperature was $230^{\circ} \mathrm{C}$ and bed temperature was $110^{\circ} \mathrm{C}$. PLA was printed using an extruder temperature of $185^{\circ} \mathrm{C}$, with a first layer bed temperature of $63^{\circ} \mathrm{C}$ to ensure adhesion followed by print bed temperature of $60^{\circ} \mathrm{C}$. Energy measurements were taken using a multimeter $( \pm 0.005$ $\mathrm{kWh}$ ) during initial heating and while printing each individual object.

The product evaluated was a juicer (Fig. 2). The juicer is used to juice oranges, grapefruits, lemons, limes and other citrus fruit. It is fixed upon a post-consumer glass jar to collect the juice. Typical manufacturing is limited on internal manipulation of a product, with the use of the RepRap, this is no longer a barrier. The fill can be altered by pattern (rectilinear, line, concentric, honeycomb, etc), angle, percentage, along with adding solid fill layers when necessary. In this study, all products were printed with the fill at 45 degrees using the rectilinear pattern and the setting of adding solid layers when necessary. The life-cycle analysis (LCA) software SimaPro 7.2 was used to get the cumulative energy demand (CED 1.07) and greenhouse gas emissions (IPCC GWP 2007 100a) for each product using the database EcoInvent v2.0. A "cradle-to-gate" analysis was done, with the gate located in the United States.

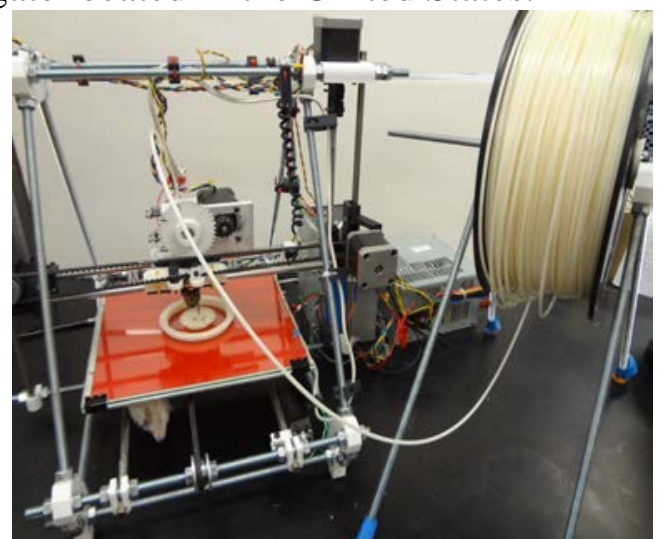

Fig. 1: Prusa Mendell RepRap 3-D printer printing a juicer using ABS.

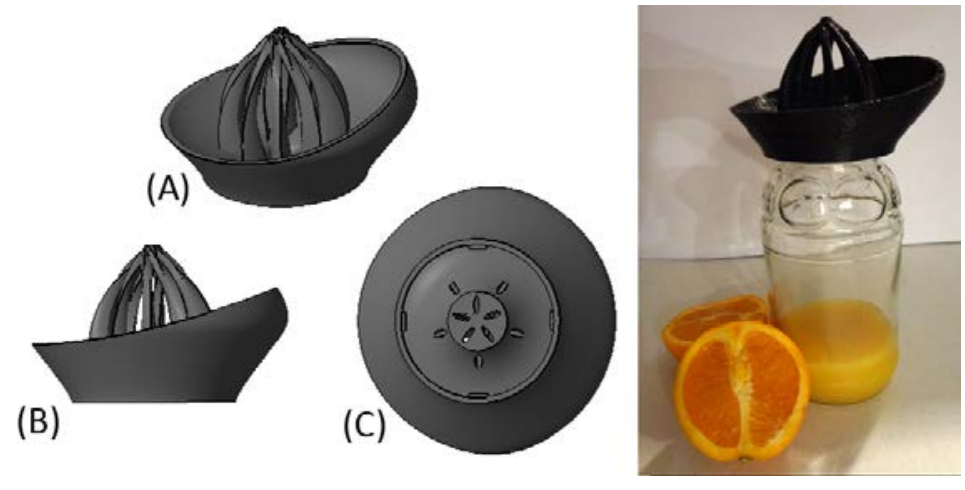

Fig. 2: Top-side (A), side (B) and bottom (C) views of CAD for a citrus juicer in photograph (STL available: http://www.thingiverse.com/thing:21100 based on original design by S. Bernier).

Distributed manufacturing was quantified using the electricity consumption of the RepRap and the material inputs by weight for ABS or PLA, where (Electricity, Production Mix, US) or (Electricity, PV, at 3kWp, a-Si panel, Switzerland) was used as an input, in addition to 
(Polylactide, NatureWorks Nebraska, US) and (Acrylonitrile-butadiene-styrene copolymer, Switzerland).

Conventional manufacturing was input into SimaPro assuming a 100\% fill and was input based on the weight from experimental results. The inputs used for conventional were material inputs PLA or ABS, as done for distributed, in addition to injection molding (Injection molding, Switzerland) and shipping 9,213 km using (Transoceanic freight ship, international) from Shanghai, China to Seattle, WA [13]. The injection molding input states that the finished product is $99.3 \%$ of its input, this was considered in the analysis for both the material input and the injection mold.

Processing for each of these cases should be assumed to underestimate the total cumulative energy demand and emissions, as additional processing may be required for consumer use (i.e. sanding, finishing, etc.). Overseas shipping distance is an underestimate due to taking a straightline trip across the ocean. Shipping over land, infrastructure, molds, packaging and waste were not included in this analysis thus underestimating the embodied energy of traditional manufacturing and are left for future work. The materials PLA and ABS were used as an example for each product, but may not be the ideal materials for these products or may require additional coatings to make them food-grade or child-safe.

\section{RESULTS AND DISCUSSION}

Measured experimental values from the RepRap are $0.31 \mathrm{kWh}$ for the PLA and $0.52 \mathrm{kWh}$ for the ABS juicers respectively. These values were input into SimaPro and compared to conventional methods as described for cumulative energy demand (CED) (Fig. 3) and greenhouse gas emissions (Fig. 4). Conventional PLA and ABS juicers at $100 \%$ fill had CED of 11.58 and 13.71 MJ, respectively. Distributed PLA and ABS juicers at $15 \%$ fill were found to have a CED of 8.66 and 12.96 MJ using conventional electricity.

The environmental impacts were minimized using a solar photovoltaic array (PV) to provide electricity following recommendations by [11] that would allow for distributed manufacturing of products with RepRap in most locations in the world. It has been well established that PV technology is a sustainable source of energy that significantly reduces environmental impact of electricity use and is amenable to distributed generation [14]. Although there are no commercial PV-powered RepRap 3-D printers, proof of concepts already exist and the open-source development community that supports the RepRap has been experimenting with variants [15]. These variants would enable distributed manufacturing even in remote communities without access to the conventional grid.

The addition of a PV system reduced the values of the CED to 6.32 and 9.03 MJ, respectively. Conventional manufacturing using PLA and ABS had the emissions of 0.43 and $0.62 \mathrm{~kg} \mathrm{CO}_{2} \mathrm{eq}$, while the distributed case with the use of conventional centralized grid electricity had 0.39 and $0.68 \mathrm{~kg} \mathrm{CO}_{2}$ eq and when powered with PV: 0.18 and $0.32 \mathrm{~kg} \mathrm{CO}_{2}$ eq. The energy is minimized using distributed manufacturing for the juicer and is made possible by using a smaller fill percentage. This not only reduces material use in the product itself but also the environmental impact of the processing and embodied energy use in the raw material extraction and transportation. The use of PV to power the RepRap minimizes both the emissions and the energy use. The emissions are lower for the distributed manufacturing systems for all cases except the ABS juicer without PV. This is due to the relatively large amount of energy needed to keep the heated build platform at operating temperature for the ABS. Future work is necessary to reduce 
the energy needed for the build platform. This can be done by using chemical means to enable better adhesion, using zoned heating so only the part of the bed that is needed for the part is heated, better insulating the bed, or using a controlled environmental chamber to insulate the entire RepRap from cold ambient temperatures.

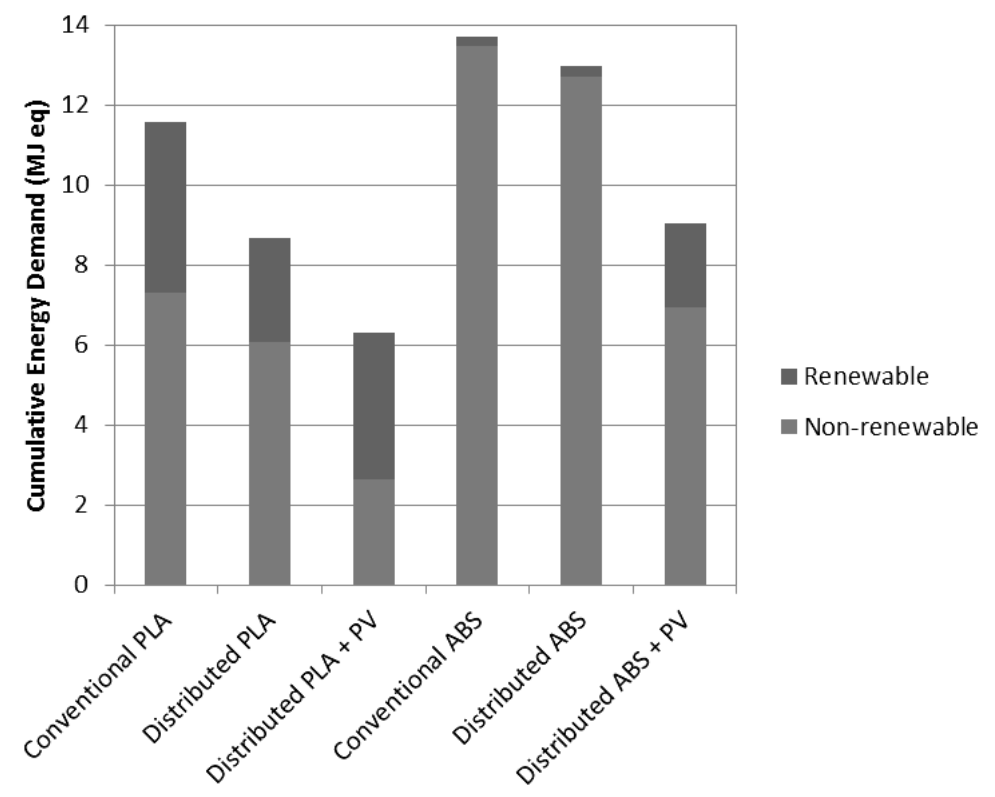

Fig. 3: CED of the juicer showing conventional PLA and ABS at 100\% fill, distributed PLA and ABS at $15 \%$ fill, along with the effect of PV electricity. Renewable consists of renewable biomass, wind, solar, and water energy sources. Non-renewable consists of non-renewable energy sources fossil fuels, nuclear, and bio-mass.

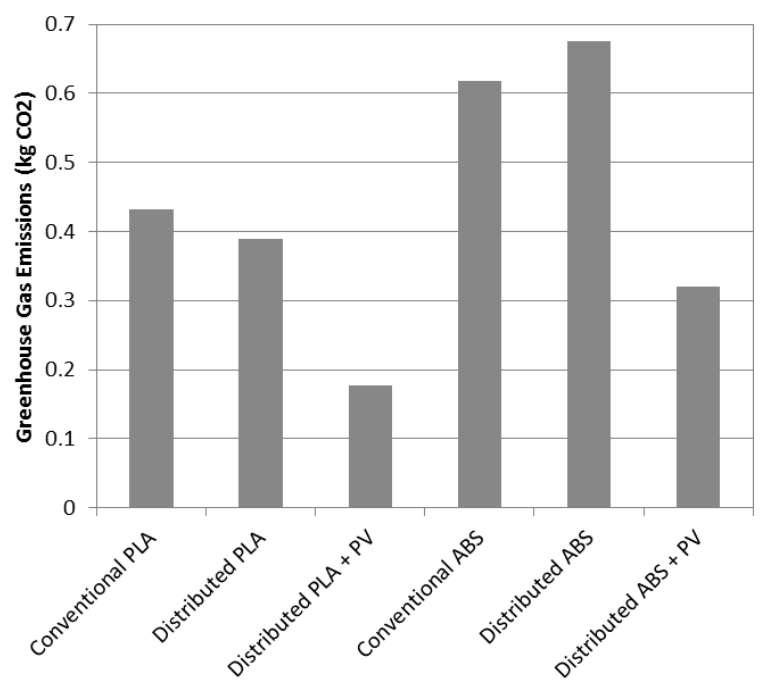

Fig. 4: Greenhouse gas emissions in $\mathrm{kg} \mathrm{CO}_{2}$ eq (GWP 100a) for the juicer for conventional PLA and ABS $100 \%$ fill, distributed PLA and ABS at 15\% fill, along with the effect of PV electricity.

The results of this study show that distributed manufacturing requires less cumulative energy than conventional manufacturing when products are made from PLA and ABS. If more products are printed simultaneously on the heating bed, it may be possible to reduce the energy 
to print even further due to the initial heating energy being dispersed among more individual products. When distributed manufacturing is used in conjunction with a PV system, the cumulative energy is further decreased. Distributed manufacturing creates fewer emissions than conventional when PLA is used and in cases using PV electricity with ABS. The emissions are highly dependent on the grid emission intensity. The addition of a PV system reduces emissions in every case and future work is needed in this area of distributed generation of electricity for distributed manufacturing. This was a preliminary study on a single product and significant future work is necessary to quantify the LCAs of distributed vs. conventional manufacturing of other products. In addition, it was clear from this preliminary study that the fill percentage plays a critical role in the environmental impact and should be systematically explored.

\section{CONCLUSIONS}

The results of this LCA study indicate that the environmental impact of distributed manufacturing with 3-D printers of polymer objects is less than conventional when using PLA. This indicates that distributed manufacturing is technically viable and environmentally beneficial because of reduced energy consumption and greenhouse gas emissions. These positive environmental results for distributed manufacturing are expanded to ABS when PV is used as a source of electricity. The preliminary results indicate that the ability of RepRaps and similar 3-D printers to vary fill percentage has the potential to significantly diminish environmental impact on many products. It can be concluded from the results of this study that open-source additive layer distributed manufacturing is both viable and beneficial from an ecological perspective.

\section{ACKNOWLEDGMENTS}

The authors would like to acknowledge helpful discussions with D.R. Shonnard, B. Tymrak and G. Anzalone. This research was supported by Sustainable Futures Institute.

\section{REFERENCES}

1. I. Kravis, R. Lipsey. J. of Int. Econ. 12(3), 201 (1982).

2. S. Thurm. Wall Street Journal. April (2012).

3. K. Ruamsook, D. Russell, E. Thomchick. J. of Supply Chain Management. 43,16 (2007).

4. R. Jones, P. Haufe, E. Sells. Robotica. 29, 1 (2011).

5. S. Upcraft, R. Fletcher. Assembly Automation. 23, 4 (2003).

6. I. Gibson, D.W. Rosen, B. Stucker. Physics Procedia. 5 (2010).

7. V. Petrovic, J.V.H. Gonzalez, O.J. Ferrando, J.D. Gordillo, J.R.B. Puchades, L.P. Griñan. Int. J. of Production Research. 49, 4 (2010).

8. A. Gebhardt, F. Schmidt, J. Hötter, W. Sokalla, P. Sokalla. Physics Procedia. 5, 2 (2010).

9. N.B. Crane, J. Tuckerman, G.N. Nielson. Rapid Prototyping Journal. 17, 3 (2011).

10. RepRap. WWW document, (reprap.org/wiki/Main_Page)

11. J. M Pearce, C. Morris Blair, K. J. Laciak, R. Andrews, A. Nosrat, I. Zelenika-Zovko, J. of Sust. Dev. 3(4), 17 (2010).

12. C. Baechler, M. DeVuono, J.M. Pearce. Rapid Prototyping. 19(2) (2013).

13. City distance calculator. WWW document (http://www.geobytes.com/citydistance.htm)

14. J. Pearce. Futures 34(7), 663 (2002).

15. D. King and J.M. Pearce. WWW document (http://www.appropedia.org/Mobile_solar_powered_3D_printer) 